Journal of Heuristics manuscript No.

(will be inserted by the editor)

\title{
Local search for the maximum $k$-plex problem
}

\author{
Wayne Pullan
}

\begin{abstract}
The maximum $k$-plex problem is an important, computationally complex graph based problem. In this study an effective $k$-plex local search (KLST) is presented for solving this problem on a wide range of graph types. KLS uses data structures suitable for the graph being analysed and has mechanisms for preventing search cycling and promoting search diversity. State of the art results were obtained on 121 dense graphs and 61 large real-life (sparse) graphs. Comparisons with three recent algorithms on the more difficult graphs show that KLS performed better or as well as in $93 \%$ of 332 significant $k$-plex problem instances investigated achieving either larger average $k$-plex sizes (including some new results) or, when these were equivalent, lower CPU requirements.
\end{abstract}

Keywords NP-complete, heuristic, local search, $k$-plex, large graphs.

\section{Introduction}

Given an undirected graph $G=(V, E)$ where $V=\left\{v_{1}, \ldots, v_{n}\right\}$ is the set of vertices and $E \subset V \times V$ is the set of edges, a $k$-plex for a given positive integer $k$ is a subset $S$ of $V$ where each vertex of $S$ is adjacent to at least $|S|-k$ vertices in the subgraph $5 \quad(S, E \cap S \times S)$. The $k$-plex problem with $k=1$ reduces to the maximum clique problem and is NP-complete [2] for all $k \geq 1$.

While there has been extensive investigation of the maximum clique problem, relatively little effort has been applied to the $k$-plex problem. However, because of the variety of real world applications for $k$-plex (including social networks, information 10 retrieval, classification theory) this is now changing and over recent time several exact algorithms 2, 8, 9, 11 have been developed. However these methods typically fail on larger problems so effort has gone into developing heuristic approaches including [4]

School of Information and Communications Technology, Griffith University, Gold Coast, Australia E-mail: w.pullan@griffith.edu.au

1 The source code for KLS is available from the author. 
which is based on the GRASP framework, a frequency based tabu search algorithm FD-TS [13], breakout local search incorporating reinforcement learning BLS-RLE [5], and reinforcement learning with configuration checking DCCplex [3].

In this paper $k$-plex local search (KLS), a new greedy/least used heuristic algorithm for the maximum $k$-plex problem is presented. Experiments show that KLS is competitive on a wide range of graph types (small-large, sparse-dense) when compared with the FD-TS, BLS-RLE and DCCplex algorithms.

The remainder of this paper is organised as follows: Section 2 presents KLS, the proposed local search for solving the $k$-plex. Section 3 contains a time and complexity analysis for KLS while Section 4 presents computational results for KLS for small and large graphs and an analysis of these results as compared to those for three other recent heuristics. Finally, a conclusion is presented in Section 5.

\section{KLS algorithm}

At a high level the KLS algorithm functions as shown in Algorithm 1 .

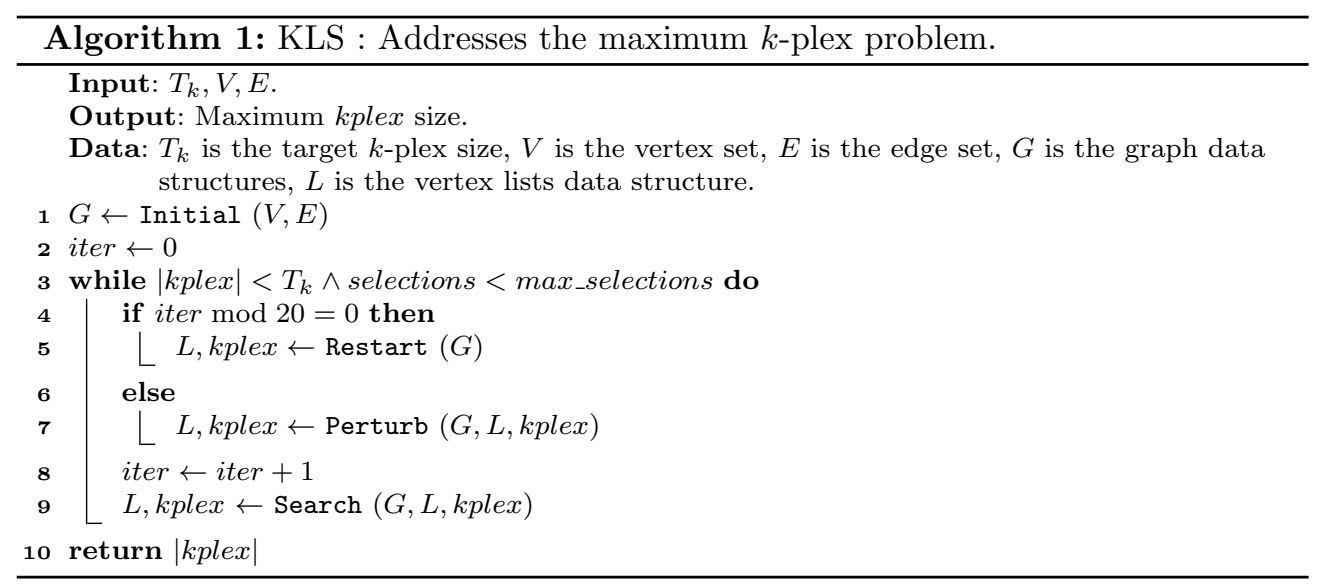

The data structures used in the KLS algorithm are:

- $G$ : Contains the vertex adjacency lists $V_{A L}$. In addition, for small graphs $(|V| \leq$ $15,000) G$ contains the vertex adjacency matrix $V_{A M}$ and, for large graphs $(|V|>$ $15,000)$, contains the $V_{O}$ data structures. These data structures are described in more detail in Section 3 .

- kplex : List of vertices currently in $k$-plex.

- $L: T_{k}+1$ vertex lists are used within $L\left(L[0], L[1], \ldots, L\left[T_{k}\right]\right)$ to partition vertices based on the number of vertices in the current $k$-plex to which they are not connected. That is, vertices in list $L[i]$ are connected to $|k p l e x|-i$ vertices in $k p l e x$. 
As discussed in Section 3, all data structures are $\mathcal{O}(1)$ for all heavily used operations and $\mathcal{O}(n)$ for infrequent operations.

The functions used in Algorithm KLS are:

- Initial $(V, E)$ : Creates $G$, the graph data structures.

$40 \quad-\operatorname{Restart}(G)$ : Creates an initial kplex containing $k$ vertices selected from the least used vertices. In an $\mathcal{O}(n)$ operation, the $L$ data structure is initialised to correctly reflect the new $k p l e x$.

- $\operatorname{Search}(G, L, k p l e x)$ : Uses the $L$ data structure and selects the least used vertex from the first non-empty list in $L[0], L[1], \ldots, L[|k p l e x|+k+1]$. This vertex is added to kplex and non-connected vertices effectively moved to the next list in $L$. If a non-connected vertex is in kplex and is now not connected to more than $k-1$ kplex vertices (i.e. is now in list $L[|k p l e x|+k+1]$ ) then it is removed from kplex. Search terminates when it has made 100 sequential additions to kplex and there has been no increase in the size of the best kplex found in this invocation of Search.

- Perturb $(G, L, k p l e x)$ : Performs a small perturbation of $k p l e x$ by removing $20 \%$ of the vertices from kplex selected from those that have been in kplex the longest.

To reduce search cycling, a circular list with five entries was associated with each vertex. These lists contain the five vertices that were added to the kplex immediately

55 prior to the last five times the associated vertex was added. If the last vertex added to $k p l e x$ is in this list then selection of the associated vertex is prohibited. This inhibits repetition of recent pair-wise sequences but allows new sequences to proceed. The time efficiency of this operation is $\mathcal{O}(1)$ and space efficiency is $\mathcal{O}(n)$.

Because Search selects the first non-empty list in $L$, the focus on least used vertices

${ }_{60}$ in Restart and Search and the removal of the oldest vertex from kplex in Perturb, KLS is a combination of a greedy and least recently used search. The greedy portion will tend towards higher degree nodes and the least used / oldest in kplex vertex selection promotes search diversity. There is no time-consuming evaluation of the impact of selecting a vertex during vertex selection.

\section{Implementation and time complexity}

A key implementation requirement for all heuristic graph algorithms is the ability to store vertex lists and graph data such that all frequently used operations are $\mathcal{O}(1)$. This was achieved in KLS as follows:

\subsection{Vertex lists}

70 KLS primarily uses an array-based implementation for vertex lists that requires two arrays of length $|V|$ and a list size variable. The first array, the list_array, starting at index zero, contains an unordered list of the vertices currently in the list. The second array, the index_array, is indexed by vertex and each entry is either the index 
of the vertex in list_array or -1 if the vertex is not in list_array. With this arraybased implementation of lists, all add (add to end of list in list_array, increment size variable, and update index_array), remove (move last vertex in list_array over vertex being removed, decrement size variable, and update index_array), and search (check index_array) operations are $\mathcal{O}(1)$ with space efficiency $\mathcal{O}(n)$. The clear operation used by Restart for all lists in $L$ is $\mathcal{O}(n)$ is implemented efficiently by iterating through each $v$ in data_array and resetting the index_array at index $v$ to -1 . The $k p l e x$ list was implemented using the array-based implementation for all graphs.

\section{$3.2 L$ Data Structure}

For small graphs, the $T_{k}+1$ vertex lists in $L$ were also implemented using the arraybased list described in Section 3.1. However, for large graphs coupled with a large $T_{k}$, the memory requirements for $L$ implemented using array-based lists became excessive so, for this case, the lists in $L$ were stored using hash tables that did not allow multiple vertices with the same hash value. If adding a vertex to the hash table caused a hash value collision (i.e. there was already a vertex stored with the same hash value), the least used of the two vertices was stored in the hash table and the other vertex was no longer available until the next Restart. The size of the hash table for each list was adaptively adjusted at each Restart based on the number of hash value collisions for the hash table since the last Restart. This implementation of hash tables gives $\mathcal{O}(1)$ performance for all add, remove, and search operations.

\section{$3.3 G$ Data Structure}

95 The $V_{A M}, V_{A L}$ and $V_{O}$ components of the $G$ data structure were implemented as follows:

- Graph adjacency matrix / lists $\left(V_{A M}, V_{A L}\right)$ : For small graphs a two-dimensional adjacency matrix $V_{A M}$ was generated. For the adjacency lists $\left(V_{A L}\right)$, each list was stored in single dimensional arrays of the appropriate size with vertices stored in ascending order. Depending on which was less, they either contained a list of adjacent vertices or a list of non-adjacent vertices and, depending on the number of entries, were sequentially or binary searched when no adjacency matrix $\left(V_{A M}\right)$ was available (i.e. for large graphs) to determine if two vertices were adjacent.

- $V_{O}$ Data Structure : For large graphs, a key performance issue is being able to quickly generate lists of vertices that, for any possible $|k p l e x|$ value, could potentially be members of kplex (as the size of kplex grows, the list of vertices that are possible candidates reduces).

In KLS this performance issue was addressed as follows: for a given graph $G=$ $(V, E)$ with $v \in V$, we define:

$$
\begin{gathered}
\text { active_degree }(v)=\operatorname{degree}(v)-\text { inactive }(v) \text { and } \\
\text { inactive }(v)=\mid\{\forall u \in V-v:(v, u) \in E \wedge \text { active_degree }(u)<|k p l e x|-k\} \mid
\end{gathered}
$$


and note that $v$ is no longer active (i.e. a possible candidate for inclusion in $k p l e x$ ) when active_degree $(v)<|k p l e x|-k$. Repeatedly applying this rule at KLS start-up allows a vertex list to be created where vertices are ordered by the maximum value of $|k p l e x|$ at which each vertex is still active (active_degree $(v) \geq|k p l e x|-k$ ) and from which the required vertex lists of candidate vertices, given a $|k p l e x|$ value, can be quickly generated.

In KLS, the $V_{O}$ data structure consists of three static single dimensional arrays:

- $V_{O L}$, of length $|V|$, contains all vertices, ordered by the maximum size of the $k p l e x$ for which they are still active (active_degree $(v) \geq|k p l e x|-k)$.

- $V_{O I}$, of length $T_{k}$, is indexed by $|k p l e x|$ and contains the index in $V_{O L}$ for the starting point for the list of all possible candidate vertices for that $k p l e x$ size. That is the vertex list $V_{O L}\left[V_{O I}[|k p l e x|]\right], \ldots, V_{O L}[|V|-1]$.

- $V_{O V}$, of length $|V|$, is indexed by vertex and contains the index in $V_{O L}$ for that vertex. If $V_{O V}[v]<V_{O I}[|k p l e x|]$ then vertex $v$ is no longer a possible candidate for kplex.

Table 1 shows the CPU time overhead of generation of the $V_{O L}, V_{O I}$ and $V_{O V}$ data structures for the large graphs used in this paper.

Table $1|V|,|E|$ for the significant problems from the SNAP/DIMACS10 large graphs used in 13 (FDS-TS) and NDR large graphs including those used in 3] (DCCplex). R is the CPU time in seconds taken for KLS to generate the $V_{O}$ data structure.

\begin{tabular}{|c|c|c|c|c|c|c|c|}
\hline Problem & $|V|$ & $|E|$ & $\mathrm{R}$ & Problem & $|V|$ & $|E|$ & $\mathrm{R}$ \\
\hline $333 \mathrm{SP}$ & $3,712,815$ & $11,108,633$ & 0.15 & kron_g500-simple-logn 16 & 65,536 & $24,56,071$ & 0.02 \\
\hline audikw1 & 943,695 & $38,354,076$ & 0.05 & rgg_n_2_20_s0 & $1,048,576$ & $6,891,620$ & 0.33 \\
\hline cage15 & $5,154,859$ & $47,022,346$ & 0.14 & rgg_n_2_21_s0 & $2,097,152$ & $14,487,995$ & 0.60 \\
\hline cit-HepTh & 27,770 & 352,285 & 0.00 & rgg_n_2_21_s0 & $2,097,152$ & $14,487,995$ & 0.60 \\
\hline cit-Patents & $3,774,768$ & $16,518,947$ & 1.89 & rgg_n_2_22_s0 & $4,194,304$ & $30,359,198$ & 1.30 \\
\hline citationCiteseer & 268,495 & $1,156,647$ & 0.07 & rgg_n_2_23_s0 & $8,388,608$ & $63,501,393$ & 3.07 \\
\hline cnr-2000 & 325,557 & $2,738,969$ & 0.10 & rgg_n_2_24_s0 & $16,777,216$ & $132,557,200$ & 5.88 \\
\hline coAuthorsCiteseer & 227,320 & 814,134 & 0.05 & uk-2002 & $18,520,486$ & $261,787,258$ & 5.37 \\
\hline coAuthorsDBLP & 299,067 & 977,676 & 0.05 & web-BerkStan & 685,230 & $6,649,470$ & 0.53 \\
\hline eu-2005 & 862,664 & $16,138,468$ & 0.56 & web-Google & 875,713 & $4,322,051$ & 0.25 \\
\hline G_n_pin_pout & 100,000 & 501,198 & 0.00 & web-Stanford & 281,903 & $1,992,636$ & 0.21 \\
\hline in-2004 & $1,382,908$ & $13,591,473$ & 0.30 & wiki-Talk & $2,394,385$ & $4,659,565$ & 0.25 \\
\hline rt-retweet-crawl & $1,112,702$ & $2,278,852$ & 0.26 & soc-twitter-follows & 404,719 & 713,319 & 0.05 \\
\hline soc-digg & 770,799 & $5,907,132$ & 0.22 & socfb-uci-uni & $58,790,782$ & $92,208,195$ & 13.27 \\
\hline socfb-A-anon & $3,097,165$ & $23,667,394$ & 0.86 & soc-flixster & $2,523,386$ & $7,918,801$ & 0.54 \\
\hline socfb-B-anon & $2,937,612$ & $20,959,854$ & 0.91 & soc-lastfm & $1,191,805$ & $4,519,330$ & 0.25 \\
\hline soc-FourSquare & 639,014 & $3,214,986$ & 0.12 & soc-livejournal & $4,033,137$ & $27,933,062$ & 3.90 \\
\hline soc-gowalla & 196,591 & 950,327 & 0.05 & soc-orkut & $2,997,166$ & $106,349,209$ & 5.16 \\
\hline soc-pokec & $1,632,803$ & $22,301,964$ & 1.23 & soc-youtube-snap & $1,134,890$ & $2,987,624$ & 0.19 \\
\hline
\end{tabular}

\section{Computational Assessment}

The major objectives of the computational experiments were to evaluate the performance of KLS as compared to three recent heuristic algorithms: Frequency Driven 
Tabu Search (FD-TS) [13, Breakout Local Search with Reinforcement Learning (BLSRLE) [5] and Local Search with Dynamic-threshold Configuration Checking and Incremental Neighborhood Updating (DCCplex) [3] on all the model $k$-plex problem instances used in these three studies.

\subsection{Computational Benchmarks}

The composite benchmark used for evaluating KLS was 80 dense graphs from the 2nd DIMACS Implementation Challenge Benchmark (DIMACS2) 2] 6], 41 graphs from the BHOSLIB ${ }^{3}$ 12 collection, 47 large real-life (sparse) graphs from the Stanford Large Network Dataset Collection (SNAP) ${ }^{4}$ [7 and the 10th DIMACS Implementation Challenge Benchmark (DIMACS10) $)^{5}$, and 17 large sparse graphs from the Network Data Repository (NDR) $)^{6}[10$.

The comparative analysis of KLS performance was restricted to significant problems from these benchmarks. In this context, a significant problem is defined as one where any algorithm (FD-TS [13, BLS-RLE 5, DCCplex 3, KLS) took longer than one second average CPU time or was not $100 \%$ successful. The 185 graphs in the composite KLS benchmark combined with 4 values for $k$ gives a total of 740 problem instances of which 332 were identified as significant problems using this criteria. For KLS, 100 independent trials, each with a different random seed and a cutoff time of 100 or 1,000 CPU seconds were performed on each problem instance with $k=2,3,4,5$. Target $k$ plex sizes were obtained by taking the maximum of the "Max" $k$-plex sizes shown in [13,5,3] except where KLS had previously found a larger value.

As a basic guide for future comparisons with KLS results, all KLS experiments were performed on an Intel(R) Xeon(R) CPU E5-2620 v4 @ 2.10GHz computer that, when only executing the DIMACS Machine Benchmark 7 required 0.31 CPU seconds for r300.5, 1.92 CPU seconds for r400.5, and 7.33 CPU seconds for r500.5. This CPU benchmarking was only available for FD-TS and showed that the FD-TS CPU times should be scaled by 0.77 to make a meaningful comparison with those of KLS. This scaling was also applied to the reported BLS-RLE CPU times as in [5] FD-TS and BLS-RLE CPU times are directly compared.

\subsection{Computational Results}

Table 2 through Table 6 summarise the experimental results for KLS and the three other algorithms. Each table shows average CPU time for the successful trials, the target $k$-plex size $\left(T_{k}\right)$ and the average $k$-plex size $\left(A_{k}\right)$ over all trials (successful and

\footnotetext{
2 http://www.cs.hbg.psu.edu/txn131/clique.html

3 http://networkrepository.com/bhoslib.php

4 http://snap.stanford.edu/data/

5 http://www.cc.gatech.edu/dimacs10/downloads.shtml

6 http://networkrepository.com/

7 fdmax.c, ftp://dimacs.rutgers.edu in directory /pub/dsj/clique
} 
unsuccessful). Where $A_{k}$ was equal to $T_{k}$ only the single value for $T_{k}$ is shown otherwise $T_{k}\left(A_{k}\right)$ is listed. Normally it was the case that when KLS failed to always achieve $T_{k}$ it would find some combination of $T_{k}$ and $T_{k}-1 k$-plex sizes. In this case, as 100 trials were used for all KLS experiments, the decimal portion of $A_{k}$ corresponds to the 170 success rate for the problem instance. Results where KLS trials attained more than 2 different $k$-plex sizes are flagged in the tables and the actual distribution of values attained given. All CPU times are in seconds and $T_{k}$ values in bold are new maximum values attained by KLS.

\subsection{Comparative Results}

Issues in comparing KLS results with those obtained by the three other algorithms arise both from different computers being used and also different experimental protocols. In summary:

- FD-TS [13] - AMD Opteron 4184 computer (2.80 GHz). CPU benchmarking provided. FD-TS results are for 20 trials for each problem instance each with a processor time-limit of 180 seconds.

- BLS-RLE [5] - Dell Precision PWS690 computer (2.66GHz). No CPU benchmarking provided. BLS-RLE results are for 20 trials for each problem instance each with a processor time-limit of 180 seconds.

- DCCplex [3] - Intel Xeon E7-4830 (2.10 GHz). No CPU benchmarking provided. DCCplex results are for 25 trials for each problem instance each with a processor time-limit of 1,000 seconds. The exception is Table 6] where a processor time-limit of 100 seconds was applied to each trial.

- KLS - Intel Xeon E5-2620 (2.10GHz). CPU benchmarking provided. KLS results are for 100 trials for each problem instance each with a processor time-limit of 1,000 seconds. The decision to use 100 trials for KLS rather than 20/25 trials was based on the expectation that this choice would result in statistically more reliable results. To match the DCCplex experimental protocol for Table 6 only, a processor time-limit of 100 seconds was applied to each KLS trial.

In this paper, comparison of results was first performed by comparing the $A_{k}$ results for each algorithm on a problem instance and, where these were deemed equivalent, to then compare average CPU times (after any scaling). In detail, when comparing two algorithms, the best performing algorithm was identified using the following two criteria, applied sequentially if necessary:

1. Quality of Result - The algorithm with the higher $A_{k}$ result, provided the difference in $A_{k}$ results was greater than 0.1 .

2. Efficiency of Algorithm - The algorithm with the lower average CPU time provided that the CPU times were different by greater than $10 \%$ of the higher CPU time. For this criteria, all CPU times for FD-TS and BLS-RLE were first multiplied by 0.77 (calculated from the respective CPU performance on the DIMACS Machine Benchmark). As no CPU performance results were supplied for DCCplex, comparisons between DCCplex and KLS for BHOSLIB and DIMACS2 graphs was on the basis of the first (Quality of Result) criteria only. 
If these two criteria did not distinguish between the two algorithms then they were deemed equivalent. Using these criteria, the comparative results shown in Table 2

through Table 6 were analysed with the following results:

- Tables 2 and 3 compare KLS with BLS-RLE and DCCplex on the significant DIMACS2 problems. For these problems, KLS was the best performing algorithm for 126 of the $153(82 \%)$ problem instances and best equal for 15 of the $153(10 \%)$ problem instances.

- Table 4 compares KLS with DCCplex on significant BHOSLIB problems. For these problems, KLS was the best performing algorithm for 52 of the 63 (83\%) problem instances and best equal for 5 of the $63(8 \%)$ problem instances.

- Table 5 compares KLS with FD-TS on the significant large problems from 13 . For these problems, KLS was the best performing algorithm for 85 of the $92(92 \%)$ problem instances.

- Table 6 compares KLS with DCCplex on the significant large problems from [3]. For these problems, KLS was the best performing algorithm for 17 of the $24(71 \%)$ problem instances and equal best for the other 7 problem instances.

Table 7 shows results for KLS for other large DNR graphs while Table 8 summarises the overall results for each benchmark. Overall, using the criteria listed above, KLS was the best performing algorithm for 280 (84\%) of the 332 significant problem instances, equal best for 27 (8\%) problem instances with one of FD-TS (7), BLS-RLE (12) and DCCplex (6) being the best performing for the remaining $25(8 \%)$ problem instances. The relative high number of equivalent algorithms arise because CPU times were not published for DCCplex meaning the 'Efficiency of Algorithm' criteria could not be applied as a tie breaking mechanism. 
Table 2 Comparison of BLS-RLE, DCCplex and KLS on significant DIMACS2 problems with $\mathrm{k}=$ 2 and $\mathrm{k}=3$. The shaded entries are where KLS was not the best performing algorithm, underlined entries are where KLS was equal best and bold-face entries are new maximums.

\begin{tabular}{|c|c|c|c|c|c|c|c|c|c|c|}
\hline Problem & \multicolumn{2}{|c|}{ BLS-RLE } & $\begin{array}{c}\mathrm{k}=2 \\
\text { DCCplex } \\
T_{k}\left(A_{k}\right)\end{array}$ & . $\mathrm{CPU}$ & $\begin{array}{l}\mathrm{KLS} \\
T_{k}\left(A_{k}\right)\end{array}$ & \multicolumn{2}{|c|}{$\begin{array}{c}\text { BLS-RLE } \\
\mathrm{PU} \quad T_{k}\left(A_{k}\right)\end{array}$} & $\begin{array}{c}\mathrm{k}=3 \\
\text { DCCplex } \\
T_{k}\left(A_{k}\right)\end{array}$ & CPU & $\begin{array}{l}\mathrm{KLS} \\
T_{k}\left(A_{k}\right)\end{array}$ \\
\hline $\begin{array}{l}\text { brock } 400-1 \\
\text { brock } 400_{2} 2\end{array}$ & & & & 1 & & $\begin{array}{l}2.07 \\
2.64\end{array}$ & $\begin{array}{l}36 \\
36\end{array}$ & & $\begin{array}{l}0.47 \\
0.43\end{array}$ & $\begin{array}{l}36 \\
36\end{array}$ \\
\hline brock400_3 & 0.2 & 301 & & I 81.47 & 31 & & & & & \\
\hline brock $400 \_4$ & 23.83 & 31 । & $33(32.84)$ & $1 \quad 1.74$ & 33 & & 1 & & $1 \ldots$ & \\
\hline brock800_1 & 2.26 & 25 । & 25 & 0.52 & 25 & 9.64 & $30(29.05)$, & $30(29.16)$ & 57.22 & 30 \\
\hline brock800_2 & 1.82 & 25 & & 0.41 & 25 & 50.02 & $30(29.95)$ & & 6.51 & $\underline{30}$ \\
\hline brock800_3 & 2.28 & 25 & 25 & 0.49 & 25 & 55.72 & $30(29.95)$ & 30 & 9.62 & 30 \\
\hline brock800_4 & 51.07 & $26(25.9)$ & $26(25.6)$ & 10.88 & 26 & 1.04 & 291 & & 0.28 & $\overline{\frac{29}{9}}$ \\
\hline C1000.9 & 51.66 & $82(81.75) ।$ & $82(81.88)$ & $\begin{array}{l}1 \quad 93.24 \\
\end{array}$ & 82 & 54.11 & $96(95.55) \mathrm{I}$ & & 106.5 & $96(95.94)$ \\
\hline $\mathrm{C} 2000.5$ & 15 & $20(19.05)$ । & $20(19.68)$ & | 129.91 & $20(19.98)$ & 10.83 & $23(22.05)$ I & $23(22.2)$ । & | 206.48 & $23(22.99)$ \\
\hline C2000.9 & 76.41 & $93(91.7)$ । & I $93(92.04)$ & | 516.19 & $93(92.06)^{1}$ & 69.55 & $109(107.3)$ । & $108(107.12)$ । & | 381.67 & $109(107.85)^{2}$ \\
\hline C250.9 & 12.96 & 551 & & $\begin{array}{ll}1 & 0.19\end{array}$ & 55 & 9.86 & 651 & & I $\quad 0.84$ & 65 \\
\hline C4000.5 & 11.86 & $21(20.15)$ । & $22(20.92)$ & 83.21 & 21 & 21.17 & $24(23.3)$ । & $24(23.6)$ । & | 58.05 & 24 \\
\hline C500.9 & 2.78 & $\begin{array}{r}69 \\
\end{array}$ & & 0.82 & $\overline{69}$ & 6.72 & 81 । & & | 3.12 & 81 \\
\hline & 0.01 & $12(10.75)$ & & 0.00 & 12 & 0.01 & $12(10.95)$ & & 0.00 & 12 \\
\hline $\mathrm{t} 200-2$ & 0.02 & $24(22.25)$ & & 0.00 & 24 & 0.01 & $24(22.3)$ & & 0.00 & 24 \\
\hline $200-5$ & 0.01 & $58(57.4)^{\prime}$ & & 0.00 & 58 & 0.01 & $58(56.85)^{\prime}$ & & 0.00 & 58 \\
\hline c-fat 500 & 0.02 & $126(124.95)$ । & & 0.03 & 126 & 0.02 & $126(124.9)$ I & & 0.01 & 126 \\
\hline at $500-1$ & 0.01 & $14(12.4)$ । & & 0.00 & 14 & 0.02 & $14(12.4) \mathrm{I}$ & & 0.00 & 14 \\
\hline fat $500-2$ & 0.01 & $26(25.2)$ । & & 0.00 & 26 & 0.02 & $26(25.25)$ । & & 0.00 & 26 \\
\hline c-fat $500-5$ & 0.01 & $64(62.45)$ । & & 0.00 & 64 & 0.02 & $64(62.45)$ । & & 0.00 & 64 \\
\hline DSJC1000_5 & 13.37 & 18 & $\underline{18}$ & 1.86 & 18 & 8.47 & 21 & 21 & 1.93 & 21 \\
\hline gen400_p0.9_55 & 40.14 & 68 & & 0.78 & $\overline{68}$ & 8.13 & $87(86.2)$ & & 0.23 & 87 \\
\hline gen 400 _p $0.9 \_65$ & 44.32 & $74(72.75) !$ & $74(73.08)$ & 146.58 & 74 & 0.46 & $101(99.35) \mathrm{I}$ & 101 & 2.04 & 101 \\
\hline gen 400 -p $0.9-75$ & 35.22 & $80(79.1)$ & & 181.78 & $80(79.99)$ & 0.19 & $114(112.5)$ । & $\underline{114} \mid$ & 0.05 & 114 \\
\hline hamming $10-2$ & 1.96 & 512 । & & I 1.96 & 512 & 2.23 & $512 \mathrm{I}$ & & 2.41 & $\overline{512}$ \\
\hline $\begin{array}{l}\text { keller5 } \\
\text { keller6 }\end{array}$ & & 63 & 63 & & & $\begin{array}{r}1.36 \\
47.53\end{array}$ & $\begin{array}{l}45 \mathrm{I} \\
93\end{array}$ & $93(9168)^{\prime}$ & $\begin{array}{r}0.29 \\
24.26\end{array}$ & $\begin{array}{l}45 \\
93\end{array}$ \\
\hline $\begin{array}{l}\text { Kellero } \\
\text { MANN_a45 }\end{array}$ & $\begin{array}{l}3.19 \\
6.61\end{array}$ & $\begin{array}{r}03 \\
662\end{array}$ & & $\begin{array}{l}0.04 \\
47.25\end{array}$ & $6 \frac{03}{62}$ & $\begin{array}{r}47.53 \\
0.84\end{array}$ & $\begin{array}{r}93 \\
990\end{array}$ & $3(91.08)$ & $\begin{array}{r}4.20 \\
0.18\end{array}$ & $\begin{array}{r}93 \\
990\end{array}$ \\
\hline MANN_a81 & 12.33 & 2162 & 2162 & 216.02 & 2162 & 31.30 & $3240 \mathrm{I}$ & 3240 & $\begin{array}{l}2.10 \\
2.34\end{array}$ & 3240 \\
\hline p_hat1500-1 & 0.33 & 14 & & 2.03 & 14 & 6.56 & 17 & & 2.07 & 17 \\
\hline p_hat $1500-2$ & 0.95 & 80 & 80 & 1.36 & 80 & & & & & \\
\hline & 50.68 & $18(16.75) \mid$ & & 400.37 & $18(17.28)$ & 3.72 & $25(23.4)^{\prime}$ & & 0.68 & 25 \\
\hline & & & & । & & 0.76 & $46(45.7)$ । & & 2.45 & 46 \\
\hline & & & & 1 & & 0.02 & $125(122.5)$ । & & 0.00 & 125 \\
\hline & 2.03 & $71(69.35)$ । & & 0.03 & 71 & 0.01 & 105(102) । & & 0.0 & 105 \\
\hline 0_0.9_3 & 7.16 & 54 & & 0.99 & 54 & 11.02 & $73(69.5)$ & & 0.02 & 73 \\
\hline $0.5 \_1$ & 9.08 & $15(14.9)$ & & 0.14 & 15 & 24.10 & $22(20.75)$ & & 0.75 & 22 \\
\hline san400_0.7_1 & 10.27 & $42(41.55)$ । & & ' 301.57 & $42(41.89)$ & 60.11 & $61(60.9)$ I & & 1.62 & 61 \\
\hline san400_0.7_2 & 36.36 & $33(32.45)$ । & 32 & | 383.45 & $33(32.07)$ & 38.19 & $47(46.8)$ I & $47(46.36) !$ & $\begin{array}{l}149.35 \\
\end{array}$ & 47 \\
\hline san400_0.7_3 & 66.1 & $28(27.75)$ । & 27 & | 347.51 & $28(27.87)$ & 21.77 & $39(37.35) ।$ & $39(38.08)$ । & | 379.23 & $39(38.59)$ \\
\hline $\operatorname{san} 400 \_0.9 \_1$ & 39.24 & 103(102.6) । & $102.2)$ & 1451.74 & $103(102.46)$ & & & & 1 & \\
\hline $\begin{array}{l}\text { Note } 1: k \text {-plex } \\
\text { Note } 2: k \text {-plex }\end{array}$ & ize dist & ibution was' & $4 \times 91,86 \mathbf{3}$ & $\times 92,10$ & estom & & & & & \\
\hline
\end{tabular}


Table 3 Comparison of BLS-RLE, DCCplex and KLS on significant DIMACS2 problems with $\mathrm{k}=$ 4 and $\mathrm{k}=5$. The shaded entries are where KLS was not the best performing algorithm, underlined entries are where KLS was equal best and bold-face entries are new maximums.

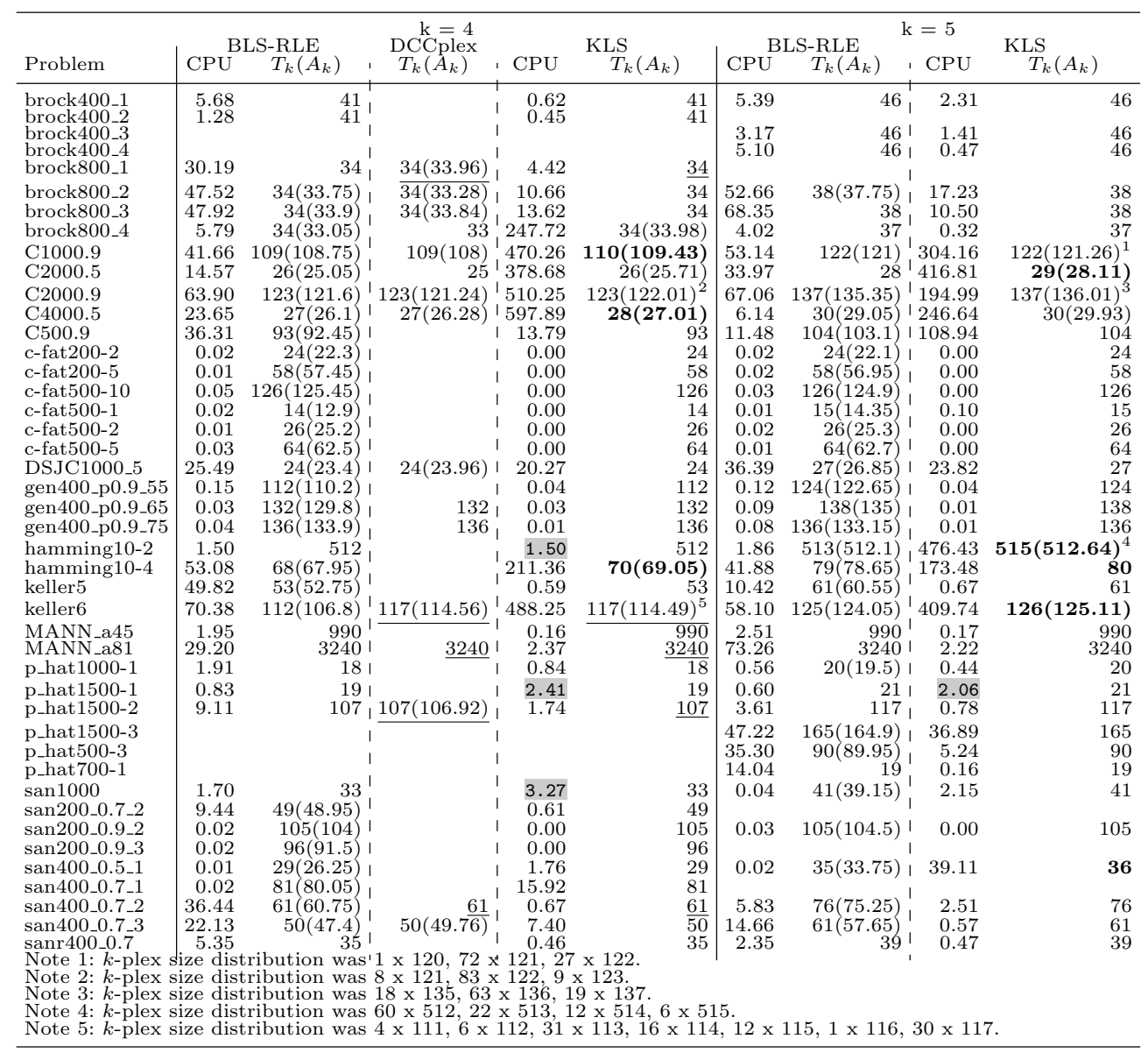


Table 4 Comparison of $T_{k}\left(A_{k}\right)$ values for DCCplex and KLS on significant problems from the BHOSLIB graphs. The shaded entries are where KLS was not the best performing algorithm, underlined entries are where KLS was equal best and bold-face entries are new maximums.

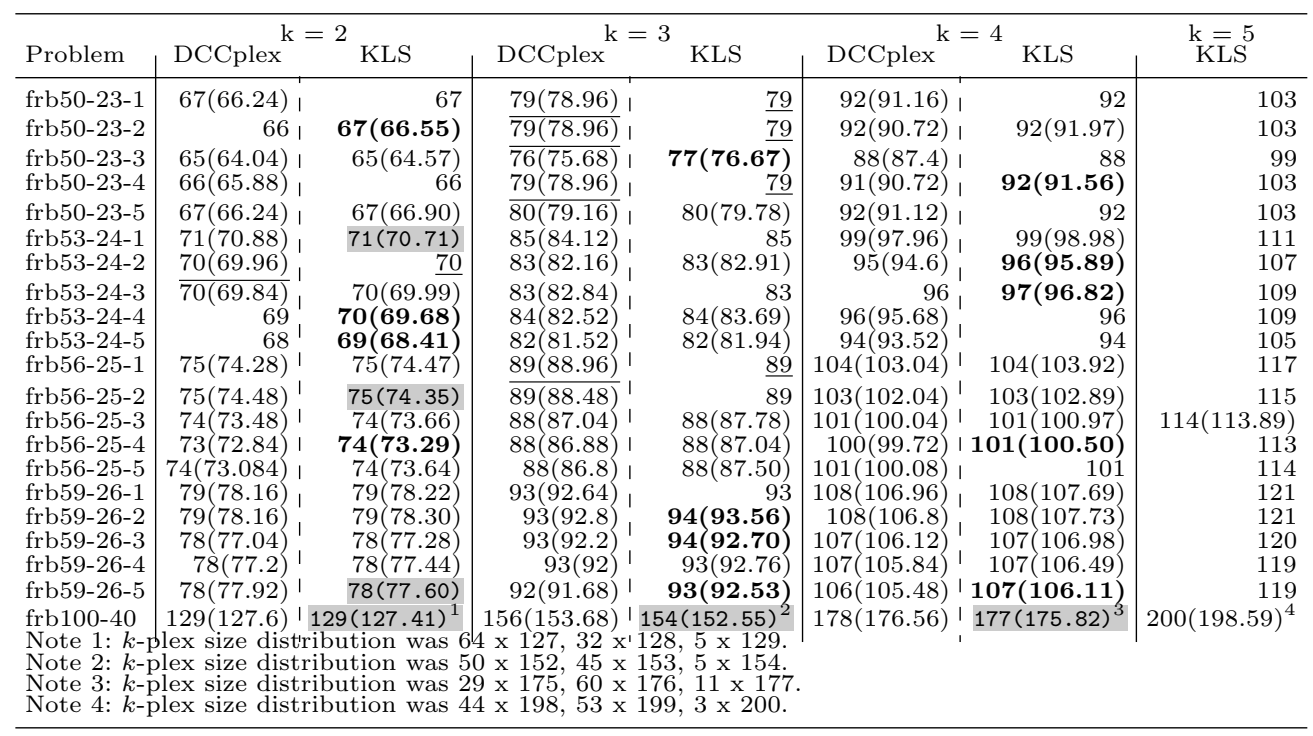

Table 5 Comparison of KLS with FD-TS on significant problems from [13. The highlighted entries are where KLS was not the best or equal best performing algorithm. $T_{k}$ is the target $k$-plex size and $A_{k}$ is the average $k$-plex size attained. FD-TS results are for 20 trials each with a CPU time-limit of 180 seconds and KLS results are for 100 trials each with a CPU time-limit of 1,000 seconds.

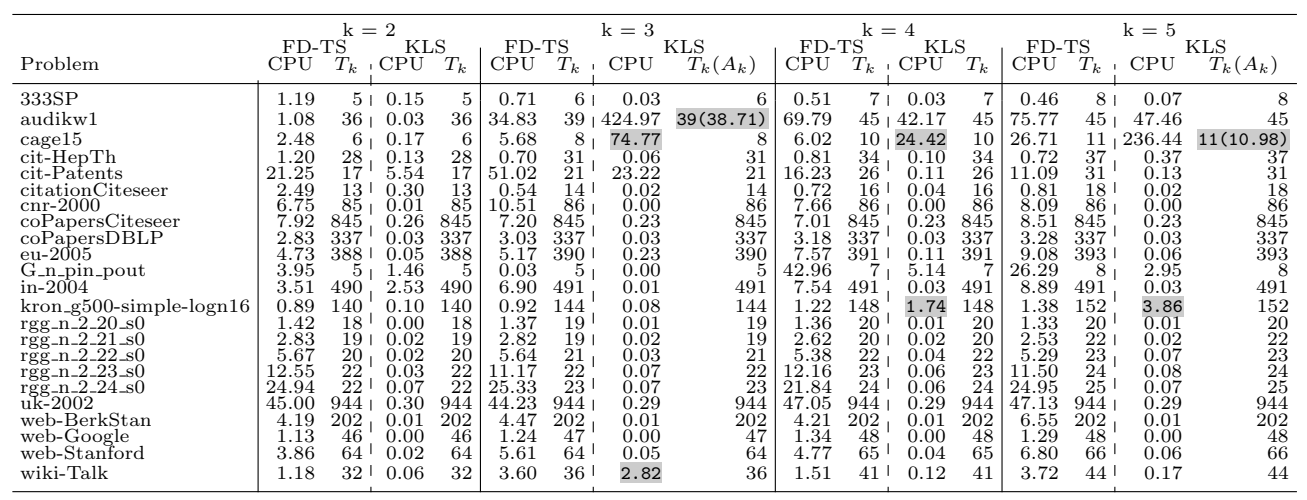


Table 6 Comparison of $T_{k}\left(A_{k}\right)$ values for KLS and DCCplex for significant DNR problems from [3]. The underlined entries are where KLS was equal best. DCCplex results are for 25 trials each with a CPU time-limit of 100 seconds and KLS results are for 100 trials each with a CPU time-limit of 100 seconds.

\begin{tabular}{|c|c|c|c|c|c|c|c|}
\hline Problem & DCCplex & $=2 \mathrm{KLS}$ & $\underset{\mathrm{DCCplex}}{\mathrm{k}}=$ & $=3 \mathrm{KLS}$ & $\frac{\mathrm{k}}{\text { DCCplex }}$ & $={ }^{4} \mathrm{KLS}$ & $\begin{array}{l}\mathrm{k} \\
\mathrm{KLS}\end{array}$ \\
\hline rt-retweet-crawl & $14(13.3)$ & 14 & $15(14.4)$ & 15 & $16(15.5)$ & 16 & 17 \\
\hline soc-digg & $57(55.3)$ & $57(56.76)$ & $63(61.8)$ & 63 & $69(68.7)$ & 69 & $72(71.45)$ \\
\hline socfb-A-anon & $28(27.3)$ & $28(27.97)$ & $32(31.2)$ & 32 & 34 & 35 & 37 \\
\hline socfb-B-anon & $27(26.6)$ & $27(26.98)$ & $30(29)$ & 30 & 33 & 33 & 35 \\
\hline soc-FourSquare & $35(34.9)$ & 35 & $39(38.7)$ & 39 & 42 & 42 & 44 \\
\hline soc-gowalla & $30(29.3)$ & 30 & 31 & 31 & $\overline{32}$ & $\overline{32}$ & 32 \\
\hline soc-pokec & $26(25.7)$ & 26 & $29(28.8)$ & $29(28.96)$ & $32(30.4)$ & $32(31.99)$ & $34(33.99)$ \\
\hline soc-twitter-follows & $\underline{8}$ & $\underline{8}$ & $\underline{9}$ & $\underline{9}$ & 11 & $\underline{11}$ & 13 \\
\hline
\end{tabular}

Table 7 Results for KLS for larger DNR problems. $T_{k}$ is the target $k$-plex size. KLS results are for 100 trials each with a CPU time-limit of 1,000 seconds.

\begin{tabular}{|c|c|c|c|c|c|c|c|c|}
\hline Problem & $\mathrm{CPU}^{\mathrm{k}}$ & $\begin{array}{l}=2 \\
T_{k}\left(A_{k}\right)\end{array}$ & $\mathrm{CPU}^{\mathrm{k}}$ & $\begin{array}{l}=3 \\
T_{k}\left(A_{k}\right)\end{array}$ & $\mathrm{CPU}^{\mathrm{k}}$ & $\begin{array}{l}\bar{T}_{k}\left(A_{k}\right) \\
\end{array}$ & $\mathrm{CPU}^{\mathrm{k}}$ & $\begin{array}{l}=5 \\
T_{k}\left(A_{k}\right)\end{array}$ \\
\hline socfb-uci-uni & 18.54 & 9 & 11.35 & 10 & 1.85 & 11 & 3.92 & 13 \\
\hline soc-flixster & 0.19 & 38 & 0.27 & 42 & 0.09 & 46 & 0.16 & 49 \\
\hline soc-lastfm & 0.14 & 18 & 0.07 & 21 & 0.05 & 24 & 1.53 & 27 \\
\hline soc-livejournal & 0.01 & 214 & 0.01 & 214 & 0.01 & 214 & 0.01 & 214 \\
\hline soc-orkut & 67.97 & 52 & 157.79 & $59(58.99)$ & 12.83 & 61 & 7.09 & 63 \\
\hline soc-youtube-snap & 0.05 & 20 & 0.02 & 21 & 0.15 & 24 & 0.10 & 26 \\
\hline
\end{tabular}

Table 8 Summary comparison of FD-TS, BLS-RLE, DCCplex and KLS for the significant problems selected from the DIMACS2, FRB, SNAP, DIMACS10 and NDR benchmarks. Entries in the table show the number of problems for which the algorithm was judged the best and, in brackets, equal best using the 'Quality of Result' and 'Efficiency of Algorithm' criteria.

\begin{tabular}{l|rrrr}
\hline Benchmark & FD-TS & BLS-RLE & DCCplex & \multicolumn{1}{c}{ KLS } \\
\hline DIMACS 2 Tables 2 and 3 & & $12(0)$ & $0(15)$ & $126(15)$ \\
FRB (Table 45 & & & $6(5)$ & $52(5)$ \\
SNAP/DIMACS 10 (Table 5 & $7(0)$ & & $0(7)$ & $85(0)$ \\
NDR (Table 6) & $7(0)$ & $12(0)$ & $6(27)$ & $280(27)$ \\
Total & & &
\end{tabular}




\section{Conclusion}

In this paper KLS, a new heuristic algorithm for the maximum $k$-plex problem was presented. KLS is effective on a wide range of graph types and uses data structures 235 suitable for the graph being analysed and has mechanisms for preventing search cycling and promoting search diversity. State of the art results were obtained on 47 large real-life (sparse) graphs from the SNAP collection and the 10th DIMACS Challenge (DIMACS10), 80 dense graphs from the 2nd DIMACS Challenge (DIMACS2), 41 graphs from the BHOSLIB collection and 14 large graphs from the Network Data

240 Repository (NDR). Comparisons with three recent algorithms (FD-TS, BLS-RLE and DCCplex) show that KLS performed better or as well as in $93 \%$ of 332 significant problem instances investigated with either larger average $k$-plex sizes (including some new results) or, when these were equivalent, lower CPU requirements.

\section{References}

1. Bader, D.A., Meyerhenke, H., Sanders, P., Wagner, D. (eds.): Graph Partitioning and Graph Clustering, 10th DIMACS Implementation Challenge Workshop, Georgia Institute of Technology, Atlanta, GA, USA, February 13-14, 2012. Proceedings, Contemporary Mathematics, vol. 588.

American Mathematical Society (2013). DOI 10.1090/conm/588. URL https://doi.org/10. 1090/conm/588

250 2. Balasundaram, B., Butenko, S., Hicks, I.: Clique relaxations in social network analysis: The maximum k-plex problem. Operations Research 59(1), 133-142 (2011)

3. Chen, P. Wan, H., Cai, S., Li, J., Chen, H. Local search with dynamic-threshold configuration checking and incremental neighborhood updating for maximum k-plex problem. In: Proceedings of the 34th AAAI conference on artificial intelligence, pp. 998-999 (2020)

4. Gujjula, K., Seshadrinathan, K., Meisami, A.: A hybrid metaheuristic for the maximum k-plex problem. Examining Robustness and Vulnerability of Networked Systems pp. 83-92 (2014)

5. Jin, Y., Drake, J.H., Benlic, U., He, K.: Effective reinforcement learning based local search for the maximum k-plex problem. CoRR abs/1903.05537 (2019). URL http://arxiv.org/abs/ 1903.05537

260 6. Johnson, D., Trick, M. (eds.): Cliques, Coloring and Satisfiability: Second DIMACS Implementation Challenge, DIMACS Series, vol. 26. American Mathematical Society (1996)

๓7. Leskovec, J., Krevl, A.: SNAP Datasets: Stanford large network dataset collection. http://snap. stanford.edu/data (2014)

8. McClosky, B., Hicks, I.: Combinatorial algorithms for the maximum k-plex problem. Journal of

265 9. Moser, H., Niedermeier, R., Sorge, M.: Exact combinatorial algorithms and experiments for finding maximum k-plexes. Journal of combinatorial optimization 24(3), 347-373 (2012)

10. Rossi, R.A., Ahmed, N.K.: The network data repository with interactive graph analytics and visualization. In: AAAI (2015). URL http://networkrepository.com

270 11. Trukhanov, S., Balasubramaniam, C. Balasundaram, B., Butenko, S. Algorithms for detecting optimal hereditary structures in graphs, with application to clique relaxations. Computational Optimization and Applications 56(1), 113-130 (2013)

12. Xu, K., Boussemart, F., Hemery, F., Lecoutre, C.: A simple model to generate hard satisfiable instances. CoRR abs/cs/0509032 (2005). URL http://arxiv.org/abs/cs/0509032

275 13. Zhou, Y., Hao, J.K.: Frequency-driven tabu search for the maximum s-plex problem. Computers and Operations Research 86, 65-78 (2017) 